\title{
Stiffness of fluid and gel phase lipid nanovesicles: weighting the contributions of membrane bending modulus and luminal pressurization
}

\section{Authors:}

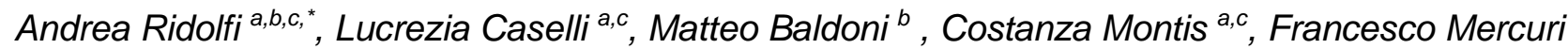

${ }^{b}$, Debora Berti ${ }^{a, c}$, Francesco Valle ${ }^{a, b,{ }^{*}}$ and Marco Brucale ${ }^{a, b,{ }^{*}}$.

a Consorzio Interuniversitario per lo Sviluppo dei Sistemi a Grande Interfase, 50019 Firenze, Italy.

${ }^{b}$ Consiglio Nazionale delle Ricerche, Istituto per lo Studio dei Materiali Nanostrutturati, 40129 Bologna, Italy.

c Dipartimento di Chimica "Ugo Schiff", Università degli Studi di Firenze, 50019 Firenze, Italy.

* corresponding authors

\begin{abstract}
The mechanical properties of biogenic membranous compartments are thought to be relevant in numerous biological processes; however, their quantitative measurement remains challenging for most of the already available Force Spectroscopy (FS)-based techniques. In particular, the debate on the mechanics of lipid nanovesicles and on the interpretation of their mechanical response to an applied force is still open. This is mostly due to the current lack of a unified model being able to describe the mechanical response of gel and fluid phase lipid vesicles and to disentangle the contributions of membrane rigidity and luminal pressure. In this framework, we herein propose a simple model in which the contributions of membrane rigidity and luminal pressure to the overall vesicle stiffness are described as a series of springs; this approach allows estimating the two contributions for both gel and fluid phase liposomes. Atomic Force Microscopy-based FS (AFM-FS), performed on both vesicles and Supported Lipid Bilayers (SLBs), is exploited for obtaining all the parameters involved in the model. Moreover, the use of coarse-grained full-scale molecular dynamics simulations allowed for better understanding the differences in the mechanical responses of gel and fluid phase bilayers and supported the experimental findings. Results suggest that the pressure contribution is similar among all the probed vesicle types; however, it plays a dominant role in the mechanical response of lipid nanovesicles presenting a fluid phase membrane, while its contribution becomes comparable to the one of membrane rigidity in nanovesicles with a gel phase lipid membrane. The herein presented results offer a simple way to quantify two of the most important parameters in vesicular nanomechanics, and as such represent a first step towards a currently unavailable, unified model for the mechanical response of gel and fluid phase lipid nanovesicles.
\end{abstract}

\section{Introduction}

Lipid membranes are fundamental components of most biological systems, delimiting the inner and outer compartments of cells, organelles and viruses, hosting a significant portion of an organism's interactome, and constituting a critical component of Extracellular vesicles (EVs) ${ }^{1}$. Several key biological processes were revealed to be affected by the mechanical characteristics of involved 
membranous compartments, including e.g. exo/endocytosis, trafficking and in some cases pathological onset ${ }^{2-5}$. Given this central role, the scientific community has devoted extended efforts for better understanding the mechanics of synthetic nanovesicles and their membranes, which represent widely used mimics for the study of biogenic membrane-bound organelles.

To this end, Force Spectroscopy (FS) techniques such as micropipette aspiration ${ }^{6}$, electrodeformation ${ }^{7}$, optical tweezers ${ }^{8}$ and Atomic Force Microscopy (AFM) ${ }^{9,10}$ are often employed, as they allow probing the mechanical properties at the nanoscale with high accuracy ${ }^{11}$. However, since it is difficult to identify and disentangle the contributions of all the parameters involved at this length scale, studying the mechanics of nanosized objects is still challenging for most of the abovementioned techniques. In particular, the accurate determination of the nanomechanical characteristics of natural and synthetic lipid vesicles with sizes $<500 \mathrm{~nm}$ remains a largely open issue, hindering multiple research fields.

AFM-based Force Spectroscopy (AFM-FS) has been recently applied for studying the mechanics of both natural and synthetic lipid vesicles ${ }^{12-15}$. The main advantage offered by AFM-FS is the possibility to simultaneously determine the exact morphology and mechanical properties of individual vesicles. In a typical AFM-FS experiment, the forces experienced by the tip during the indentation of a vesicle are recorded as a function of the tip-sample separation; these data are then plotted as force versus distance curves. According to the Canham-Helfrich theory (CHT) 16,17 , the initial mechanical response of a lipid vesicle to indentation is elastic and follows a linear correlation between the applied perpendicular force and the penetration depth. The resulting mechanical response can be broadly described in terms of Hooke's law $F=-K x$, where $K$, the stiffness $(K)$ of the vesicle, can be estimated from the slope of the observed linear regime. Stiffness is an extensive property resulting from multiple contributions, the most important of which are the intrinsic membrane elasticity and the luminal pressure, the latter being defined as the internal pressure that originates from the fluid confined within a vesicle.

In order to quantify a vesicle's membrane intrinsic elasticity, its contribution to the experimentally accessible quantity $K$ has to be disentangled from the others. Among the various biophysical descriptors, the bilayer bending modulus, $(\mathrm{K})$ is a widely used parameter in membrane biophysics to quantify the energy required to deform a membrane from its spontaneous curvature ${ }^{18}$; moreover, its evaluation is of fundamental importance for understanding the effect of the membrane bending rigidity in biological processes like vesicle fusion and budding.

Several theories and models have been proposed in the AFM-FS literature to derive $\mathrm{k}$ from the measured $K$ values, obtaining different degrees of agreement with the results from other techniques (examples can be found in Table 1). The description is further complicated by the dual nature that lipid membranes display above and below their melting temperature $\left(T_{m}\right)$; at $T>T_{m}$, lipid bilayers are generally found in the so-called fluid phase, in which their acyl chains present an increased lateral mobility compared to the case of $\mathrm{T}<\mathrm{T}_{\mathrm{m}}$, where membranes display the so-called gel phase, 
characterized by a limited lateral mobility and a tighter packing degree between the acyl chains of the two leaflets. One of the most straightforward theories used to describe the mechanics of adsorbed lipid vesicles is the Thin Shell Theory (TST) ${ }^{19}$; which models the lipid vesicle as being solely constituted by a homogeneous shell of thickness $h$ and curvature radius $R$, provided that the ratio $h / R$ is sufficiently small ${ }^{20}$. Following this approach, TST does not account for the luminal pressurization, hence ascribing all the energetic contributions of vesicle indentation to the membrane elasticity. Moreover, by describing the membrane as a single homogeneous shell, simple TST models ignore the fact that in fluid phase bilayers, the two leaflets are free to slide upon each other. Reissner et al. ${ }^{21,22}$ generalized the TST and proposed an analytical solution for the case of shallow segments of thin elastic spherical shells, which takes into consideration the presence of transverse shear deformations ${ }^{20}$.

More recently, Vorselen et al. ${ }^{23,24}$ found that the pressure contribution to the indentation response of nanosized fluid phase liposomes accounts for a great part of the overall deformation energy. Based on these findings, they developed a CHT-based model that allows calculating both the bending modulus and the luminal pressure of nanosized fluid phase lipid vesicles from their stiffness $K$ and tether force (i.e. the force at which a lipid tube of uniform diameter is elongated away from the vesicle by the AFM tip). This model has been employed for studying the mechanical properties of $\mathrm{EVs}^{25}$, revealing that specific pathological conditions can induce a change in their membrane rigidity 4.

In this context, it is immediately apparent that the two above-presented models differ irreconcilably in their treatment of the lipid bilayer, which is modeled either as a single homogeneous shell in one case (TST) or as a pair of independently sliding monolayers in the other. These different scenarios seem at first glance most suited to respectively model vesicles constituted by lipids in their gel and fluid phases, thus suggesting that the applicability of the two models might be dictated by the state of the lipid membrane under investigation.

An orthogonal experimental strategy to determine via AFM-FS the $\mathrm{k}$ values of membranes, is to drastically simplify the problem and deposit them on a rigid substrate obtaining Supported Lipid Bilayers (SLBs) ${ }^{26,2728}$, whose indentation mechanics is considerably simpler to model with respect to intact vesicles. This is mainly due to the fact that the mechanical response of SLBs is not affected by internal pressure-related phenomena, hence making it possible to univocally relate the SLB indentation forces to the rigidity of the bilayer. To this purpose, various contact mechanics models have been developed to extract $\mathrm{k}$ from AFM-FS experiments on SLBs ${ }^{9}$. Despite the extensive number of reports both on SLBs and vesicles, there is still disagreement between the $\mathrm{k}$ values measured on the same membranes in the two experimental configurations. This issue further complicates the interpretation of experimental data and ultimately, hinders a complete understanding of several membrane-related processes in terms of stiffness. 
In the attempt to reconcile the different interpretations outlined above, we propose a simple model where the contributions of membrane rigidity and vesicle luminal pressure to the overall stiffness of a nanosized vesicle are described as a series of springs. This approach permits to quantitatively estimate the individual contributions to the stiffness of fluid or gel phase nanosized vesicles by using a single model. We test this approach on a library of synthetic liposomes, composed of phospholipids having the same polar head group (phosphatidylcholine) but different acyl chains. This allows exploring different lipid lateral interaction energies, spanning the bilayer phase space between fluid and gel phases. All the bilayers are probed by AFM-FS both as SLBs and vesicles, allowing us to quantitatively distinguish the contributions of the membrane bending modulus and luminal pressure to the overall stiffness of the liposomes. Particle-based simulations performed on realistic models of lipid bilayers mimicking the experimental set-up are then employed to support the AFM-FS results and provide new insights on the origin of the observed different mechanical responses.

\section{Materials and Methods}

\section{Vesicles preparation}

Different lipids with PC polar headgroup (DOPC (1,2-dioleoyl-sn-glycero-3-phosphocholine) (>99\%), POPC (1-palmitoyl-2-oleoyl-glycero-3-phosphocholine) ( $\geq$ 98.0\%), DPPC (1,2-dipalmitoyl-snglycero-3-phosphocholine) (>99\%), DSPC (1,2-1,2-distearoyl-sn-glycero-3-phosphocholine) $(>99 \%)$ ) were purchased from Sigma Aldrich (St. Louis, MO, USA). All chemicals were used as received. Lipid dry powders were dispersed in defined amounts of chloroform, to prepare stock solutions. Lipid films were obtained by evaporating appropriate amounts of lipid stock solutions in chloroform under a stream of nitrogen, followed by overnight drying under vacuum. The films were swollen by suspension in warm $\left(50^{\circ} \mathrm{C}\right.$ ) water (Milli-Q grade water was used in all preparations) to a final lipid concentration of $4 \mathrm{mg} / \mathrm{mL}$, followed by vigorous vortex mixing. The resultant multilamellar liposomes in water were subjected to 10 freeze-thaw cycles and extruded 10 times through two stacked polycarbonate membranes with $100 \mathrm{~nm}$ pore size at room temperature, to obtain unilamellar liposomes with narrow and reproducible size distribution. The filtration was performed with the Extruder (Lipex Biomembranes, Vancouver (Canada)) through Nuclepore membranes (please refer to Caselli et al. ${ }^{29}$ for further details about the vesicle preparation and characterization).

\section{Surface cleaning procedure}

All reagents were purchased from Sigma-Aldrich Inc (www.sigmaaldrich.com). Liposomes and supported lipid bilayers were measured on microscopy borosilicate glass coverslips (Menzel Gläser) and on $\mathrm{SiO}_{2}$ wafers, respectively. Surfaces were first immersed in a 3:1 mixture of $96 \% \mathrm{H}_{2} \mathrm{SO}_{4}$ and $30 \% \mathrm{v} / \mathrm{v}$ aqueous $\mathrm{H}_{2} \mathrm{O}_{2}$ ('oxidizing piranha') solution for $2 \mathrm{~h}$ in order to remove any organic residue present on their surface. Surfaces were then cleaned in a sonicator bath (Elmasonic Elma S3OH) for 
30 minutes in acetone, followed by 30 minutes in isopropanol and 30 minutes in ultrapure water (Millipore Simplicity UV). After this procedure, substrates can be stored in ultrapure water preserving their pristine conditions for weeks.

\section{Surface preparation for AFM-FS on intact vesicles}

Cleaned glass coverslips were treated with air plasma for 15 minutes (Air plasma cleaner PELCO easiGlow) and incubated overnight in ultrapure water in order to maximize silanol surface density. Slides were then functionalized by vapor-phase silanization with (3-Aminopropyl)triethoxysilane (APTES). Small batches of 3-5 slides were put in a desiccator with $30 \mu \mathrm{l}$ of APTES and $10 \mu \mathrm{l}$ of triethylamine (TEA), then a gentle static vacuum was induced by briefly engaging a rotary pump. Glass slides were then kept in these conditions for 8 hours. TEA was used to promote APTES -silanol binding ${ }^{30}$. After that, functionalized glass coverslips can be stored in sealed petri dishes, preserving the same surface properties for several weeks (please see SI for surface characterization).

\section{Surface preparation for AFM-FS on SLBS}

Cleaned silicon wafers were treated with air plasma for 15 minutes (Air plasma cleaner PELCO easiGlow), incubated in ultrapure water for 10 minutes in order to maximize the number of reactive surface silanols, then dried with nitrogen.

\section{SLB formation via vesicle fusion}

A $100 \mu \mathrm{l}$ droplet of $200 \mathrm{mM} \mathrm{CaCl}_{2}$ diluted 1:10 in $100 \mathrm{mM} \mathrm{NaCl}$ was spotted on a $\mathrm{SiO}_{2}$ slide. A $10 \mu \mathrm{l}$ droplet of the chosen vesicular dispersion was then added to the previous droplet and left incubating at room temperature for 30 minutes in order to promote vesicle adsorption on the surface. After that, the droplet was removed via nitrogen flow and replaced by a $100 \mu$ ldroplet of ultrapure water which was then left incubating for additional 15 minutes. After the system equilibrated, the large droplet was gently removed, and the slide placed in the AFM fluid cell for the measurements. This procedure is reported to promote the formation of continuous and homogeneous SLBs ${ }^{31}$.

\section{AFM setup}

All AFM experiments were performed on a Bruker Multimode8 (equipped with Nanoscope $V$ electronics, a sealed fluid cell and a type JV piezoelectric scanner) using Bruker SNL-A probes (triangular cantilever, nominal tip curvature radius 2-12 nm, nominal elastic constant $0.35 \mathrm{~N} / \mathrm{m}$ ) calibrated with the thermal noise method ${ }^{32}$. The temperature within the fluid cell was $28^{\circ} \mathrm{C}$.

\section{AFM imaging}

Imaging was performed in PeakForce mode. In order to minimize vesicle deformation or rupture upon interaction with the probe, the applied force setpoint was kept in the $150-250 \mathrm{pN}$ range. Lateral 
probe velocity was not allowed to exceed $5 \mu \mathrm{m} / \mathrm{s}$. Feedback gain was set at higher values than those usually employed for optimal image quality in order to ensure minimal probe-induced vesicle deformation upon lateral contact along the fast scan axis (a comprehensive explanation of this procedure was given elsewhere ${ }^{33}$ ). The average height value of all bare substrate zones was taken as the baseline zero height reference. Image background subtraction was performed using Gwyddion 2.53.16 ${ }^{34}$.

\section{AFM force spectroscopy on vesicles}

In order to perform the mechanical characterization of vesicles via AFM-FS, the samples were first scanned to locate individual vesicles. The chosen vesicle was then imaged at higher resolution ( $500 \times 500 \mathrm{~nm}$ scan, $512 \times 512$ points); its height profile along the slow scan axis was fitted with a circular arc only taking into account values $10 \mathrm{~nm}$ above the bare substrate (typical fit $\mathrm{R}^{2} \geq 0.95$ ). This procedure yielded, for each vesicle, an apparent fitted curvature radius $\mathrm{R}_{\mathrm{C}}$ and a vesicle height value $\mathrm{H}$, which were corrected as described elsewhere ${ }^{23}$. To avoid intrinsic piezo inaccuracy and drift, which imply a certain degree of uncertainty on both the $X Y$ position at which the force curve is performed relative to the original image and on the maximum applied force, multiple force curves were performed. In particular, we recorded a series of force/distance curves at multiple XY positions (typically around 64-100 curves arranged in a square array covering the vesicle initial location) for each individual vesicle. In most cases, only a few curves showed the full mechanical fingerprint of an intact vesicle on both the approach and retraction cycles, showing a linear deformation upon applied pressure and a tether elongation plateau during probe retraction. Of these, we first discarded those with probe-vesicle contact points occurring at probe-surface distances below vesicle height as measured by imaging. We then discarded traces in which the tether elongation plateau occurring during probe retraction did not extend beyond the initial contact point (further details can be found in $\left.{ }^{33}\right)$. The remaining traces were analyzed to calculate vesicle stiffness $(K)$ and tether elongation force. Multiple valid curves referring to the same vesicle resulted in very narrow distributions of both $K$ and tether force (with average measured values taken as representative for each vesicle), while different vesicles of the same type showed much larger variations.

\section{AFM force spectroscopy on SLBs}

When performing AFM-FS on SLBs, the accuracy of the $X Y$ position at which each force curve is performed becomes less important in comparison to vesicles. We nevertheless recorded a series of force/distance curves at multiple XY positions (typically around 64-100 curves arranged in a square array covering large regions of the SLB) in order to minimize the impact of (putative) local anisotropies of either the substrate or the bilayer on the measured mechanical properties. The recorded curves were then analyzed to extract bending modulus $(\mathrm{K})$ values. 


\section{Particle-based molecular dynamics (MD) simulations}

Particle-based molecular dynamics (MD) simulations were performed on realistic models of SLBs using the Martini coarse-grained potential| ${ }^{35-37}$. Simulations were performed with the LAMMPS program package ${ }^{38}$ and run on the CNR-ISMN high-performance computing facility.

The model system was composed by a 2D-periodic support surface, a finite-size model of SLBs in water solution and a model of a mechanical probe. Models of DPPC and DOPC lipid bilayers in water solution were considered. Upon equilibration onto the substrate in water, lipid bilayer models relax into a round-like shape, with a diameter of about $24 \mathrm{~nm}$. A model of a mechanical probe mimicking the AFM tip was built as a disc of SG4 beads, similar to the support surface. A diameter of the AFM tip model of $18 \mathrm{~nm}$ was considered. Mechanical properties of SLB were simulated by reproducing the displacement of the AFM tip towards the surface, which was kept fixed in simulations. A first trajectory was obtained by displacing the AFM tip towards the SLB at a constant velocity of 0.1 $\mathrm{nm} / \mathrm{ns}$. This first fast trajectory allowed us to obtain starting configurations for subsequent accurate sampling of force vs. distance curves. Further details are provided in the Supplementary Information section.

\section{Results and Discussion}

We employed AFM-FS to measure the mechanical response of a series of lipid bilayers in their planar (SLB) and vesicular configurations. All the FS experiments were performed under the same experimental conditions (deposition protocol, solution, temperature, substrate; see Materials and Methods section). All the probed lipids have the same polar head group (phosphatidylcholine, PC) but differ in the length and degree of unsaturation of their hydrocarbon chains. As a general rule, short and unsaturated hydrocarbon tails generate softer lipid bilayers while long fully saturated tails have a higher packing degree, which increases the overall bilayer rigidity. It is known from the literature that the bending modulus of lipid bilayers used in this study increases in the following order: DOPC < POPC < DPPC < DSPC ${ }^{29,39,40 . ~ A l l ~ t h e ~ f o u r ~ l i p i d s ~ w e r e ~ u s e d ~ t o ~ f o r m ~ l i p o s o m e s ~ a n d ~ S L B s, ~}$ then measured via AFM-FS.

\section{Measurement of vesicle stiffness}

We first measured the stiffness of several different vesicles composed of each of the lipids in the above-mentioned set ${ }^{29,41}$. Despite stiffness being an extensive property, the very similar average size and narrow polydispersity of the measured liposomes resulted in a relatively small variance within each sample (as it can be seen from the small overlap between the error bars in Figure 1 and from their numerical values in the second column of Table 3). The liposomes follow the expected stiffness ranking, with DOPC liposomes being the softest and DSPC liposomes the stiffest, and the 
stiffness values are in close agreement with those reported in the literature for similar-sized vesicles 23,42 .

\section{Measurement of SLB bending modulus}

We then performed AFM-FS measurements on SLBs obtained from the rupture of the same set of liposomes. The mechanical response of SLBs to indentation is much simpler to model with respect to that of vesicles; indeed, their bidimensional geometry and the absence of internal pressure contributions allow to unambiguously probe the membrane rigidity in itself. Once the bilayer adsorbs on the substrate, it can be effectively modeled as a layer of a continuous material and the AFM tip can hence be used to apply a perpendicular force to the SLB, resulting in its compression. Fitting an appropriate contact mechanics model to the recorded indentation traces allows extracting quantitative nanomechanical information about the SLB. Among the numerous contact mechanics models developed to describe the indentation of a flat material by probes of various shapes and sizes $^{9}$, we found that the modified Hertz model ${ }^{43}$ proposed by Dimitriadis et al. ${ }^{44}$ is the one that best fits all our SLB indentation profiles (Figure S1). According to this model, the Young modulus of the probed bilayer can be calculated from Equation 1:

$$
F=\frac{16}{9} E R^{1 / 2} \delta^{3 / 2}\left[1+0.884 \chi+0.781 \chi^{2}+0.386 \chi^{3}+0.0048 \chi^{4}\right]
$$

where the force $F$ is related to the Young modulus $E$, tip radius $R$, penetration depth $\delta$ and $\mathrm{X}$, which is equal to $\sqrt{R \delta / h}, h$ being the thickness of the bilayer (evaluated from AFM imaging experiments on the different SLBs and in good agreement with the literature ${ }^{14,45-48}$, please refer to Figure S3 for further details). The Young modulus $E$ can hence be used to obtain the SLB bending modulus ( $\kappa_{S L B}$ ) by exploiting the Thin Shell Theory (TST) (Equation 2), where $v$ is the Poisson modulus (assumed to be $0.5^{12,14}$ for all following calculations).

$$
\kappa_{S L B}=\frac{E h^{3}}{12(1-v)}
$$

The values obtained for the bending moduli of the whole SLB series are $(19.2 \pm 2.8) k_{B} T$ for DOPC, (47.3 \pm 5.2$) k_{B} T$ for POPC, $(240.1 \pm 50.4) k_{B} T$ for DPPC and (335.8 \pm 48.1$) k_{B} T$ for DSPC; remarkably, they follow the same trend observed for the stiffness of the respective liposomes and are in good agreement with the vast majority of literature results in that fluid phase bilayers typically show bending moduli around one order of magnitude lower than those of gel phase bilayers ${ }^{49}$. Table 1 reports a comparison of our results with several bending modulus values reported in the literature both for fluid and gel phase bilayers. 
Table 1

\begin{tabular}{|c|c|c|c|c|c|}
\hline Reference & Lipid & Technique & $\begin{array}{c}\text { Temperature } \\
\left({ }^{\circ} \mathrm{C}\right) \\
\end{array}$ & $\mathrm{K}\left(\mathrm{x} 10^{19} \mathrm{~J}\right)$ & $\begin{array}{c}\mathrm{K} \\
\left(k_{B} \mathrm{~T}\right) \\
\end{array}$ \\
\hline Liu, Y., et al., $2004^{50}$ & DOPC & Scattering experiments & 30 & 0.8 & 19.1 \\
\hline Levine Z. A., et al., 2014 & DOPC & Atomistic simulations & 25 & 1.1 & 27.7 \\
\hline $\begin{array}{l}\text { Et-Thakafy, O., et } \\
\text { al.,2017 }\end{array}$ & DOPC & AFM-FS on vesicles & 25 & 0.9 & 21.9 \\
\hline $\begin{array}{l}\text { Et-Thakafy, O., et } \\
\quad \text { al.,2017 } 14\end{array}$ & DOPC & AFM-FS on SLBs & 25 & 0.9 & 21.4 \\
\hline Picas, L., et al., $2012^{52}$ & DOPC & AFM-FS on SLBs & 25 & 0.7 & 18.0 \\
\hline present study & DOPC & AFM-FS on vesicles & 28 & 0.8 & 18.7 \\
\hline present study & DOPC & AFM-FS on SLBs & 28 & 0.8 & 19.2 \\
\hline Dimova, R., et al., 2014 & POPC & X-ray scattering & 30 & 0.9 & 20.3 \\
\hline Nagle, J. F., et al., 2017 & POPC & X-ray scattering & 30 & 1.1 & 25.7 \\
\hline $\begin{array}{l}\text { Henriksen, J., et al., } \\
200655\end{array}$ & POPC & Micropipette aspiration & 25 & 1.6 & 38.5 \\
\hline present study & POPC & AFM-FS on vesicles & 28 & 1.6 & 39.3 \\
\hline present study & POPC & AFM-FS on SLBs & 28 & 2.0 & 47.6 \\
\hline $\begin{array}{l}\text { Et-Thakafy, O., et } \\
\quad \text { al.,2017 } 14\end{array}$ & DPPC & AFM-FS on SLBs & 25 & 2.0 & 49.3 \\
\hline Zheng Y., et al., $2009^{56}$ & DPPC & Neutron Spin echo & 30 & 2.1 & 49.6 \\
\hline Picas, L., et al., $2012^{52}$ & DPPC & AFM-FS on SLBs & 25 & 2.3 & 56.6 \\
\hline $\begin{array}{l}\text { Et-Thakafy, O., et } \\
\quad \text { al.,2017 } 14\end{array}$ & DPPC & AFM-FS on vesicles & 25 & 15.5 & 376.7 \\
\hline Delorme, & DPPC & AFM-FS on vesicles & 25 & 13.5 & 329.1 \\
\hline present study & DPPC & AFM-FS on vesicles & 28 & 4.7 & 113.4 \\
\hline present study & DPPC & AFM-FS on SLBs & 28 & 10.0 & 240.1 \\
\hline Zheng Y., et al., 200956 & DSPC & Neutron Spin echo & 40 & 3.4 & 79.1 \\
\hline Daillant, J., et al.,2005 49 & DSPC & X-ray scattering & $\sim 50$ & 11.2 & 275.0 \\
\hline present study & DSPC & AFM-FS on vesicles & 28 & 5.2 & 125.9 \\
\hline present study & DSPC & AFM-FS on SLBs & 28 & 14.0 & 335.8 \\
\hline
\end{tabular}

\section{Vesicle stiffness and SLB bending modulus are linearly correlated}

While the $K_{S L B}$ values obtained as described above are intensive mechanical properties specific for each bilayer type, the stiffness values $K$, measured on their vesicular configuration are extensive properties and might be influenced by size and/or geometry artifacts. Nevertheless, it is possible to obtain a strong indication of $K$ being representative of the vesicles' mechanical response by plotting it against $K_{S L B}$ for the whole series of lipids (see Figure 1). The resulting linear correlation between mechanical descriptors obtained from two series of independent measurements, performed with the 
same setup, on the same lipid bilayers, in either planar or vesicular geometry, can be considered as indicative of two facts: first, that the average stiffness $K$ is indeed a good descriptor of mechanical differences occurring across the panel of lipids, as previously hypothesized (see above, "Measurement of vesicle stiffness"). Second, that - despite $K$ being a complex parameter emerging from the interplay of several concurring phenomena including vesicle geometry, bilayer bending modulus and its resistance to pressurization - all of its determinants appear to be effectively recapitulated in just one parameter, $\kappa$.

This observation can be rationalized as follows; $K$ values were measured on vesicles having similar sizes, same polar head group, and in the absence of an osmotic imbalance; in these conditions, any systematic difference between the mechanical responses exhibited by vesicles of different composition will necessarily arise from the different degree of interaction exhibited by their constituent lipids. Different interlipidic interaction energies will ultimately determine both $k$ and the various phenomena contributing to $K$, thus resulting in the observed direct proportionality between $K_{S L B}$ and $K$. Moreover, the linear relation displayed in Figure 1 is in good agreement with Dai et al. ${ }^{58}$, who theorized a linear relation between the stiffness and the bending modulus of lipid nanovesicles.

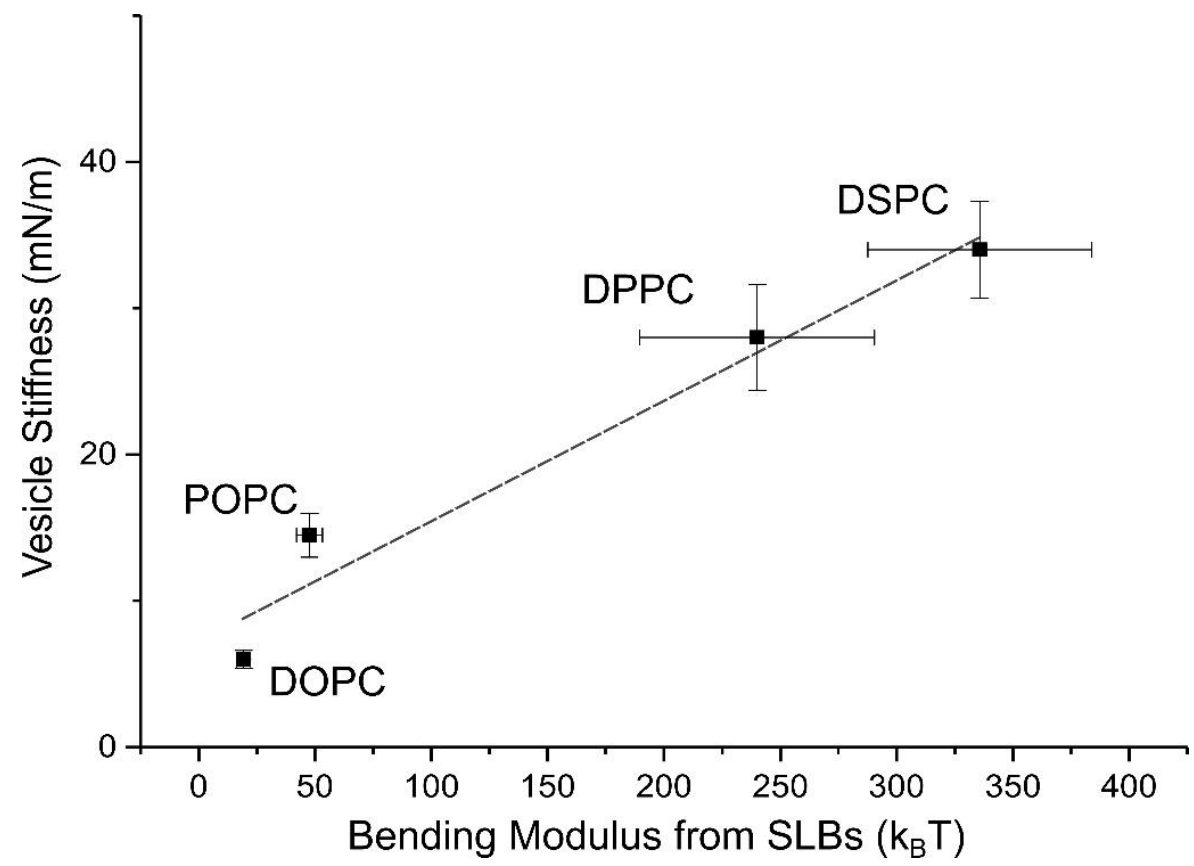

Figure 1: Linear correlation between average vesicle stiffness $K$ and average SLB bending modulus $k_{b}$. Error bars for vesicle stiffness describe the standard deviation of the mean while the ones for $k_{b}$ of SLBs represent the uncertainties obtained by bootstrapping (1000 repetitions of 5 draws, with replacement).

\section{Molecular dynamics simulations}

Coarse-grained simulations were performed on model systems of SLBs, with the aim of reproducing their response to the indentation by the AFM tip. A representative snapshot of a configuration extracted from MD simulations for the evaluation of the mechanical properties of SLBs is shown in 
Fig. S2 (see also the Computational Details section). The two considered lipids, DOPC and DPPC, are representative of the fluid and gel state of SLBs, respectively.
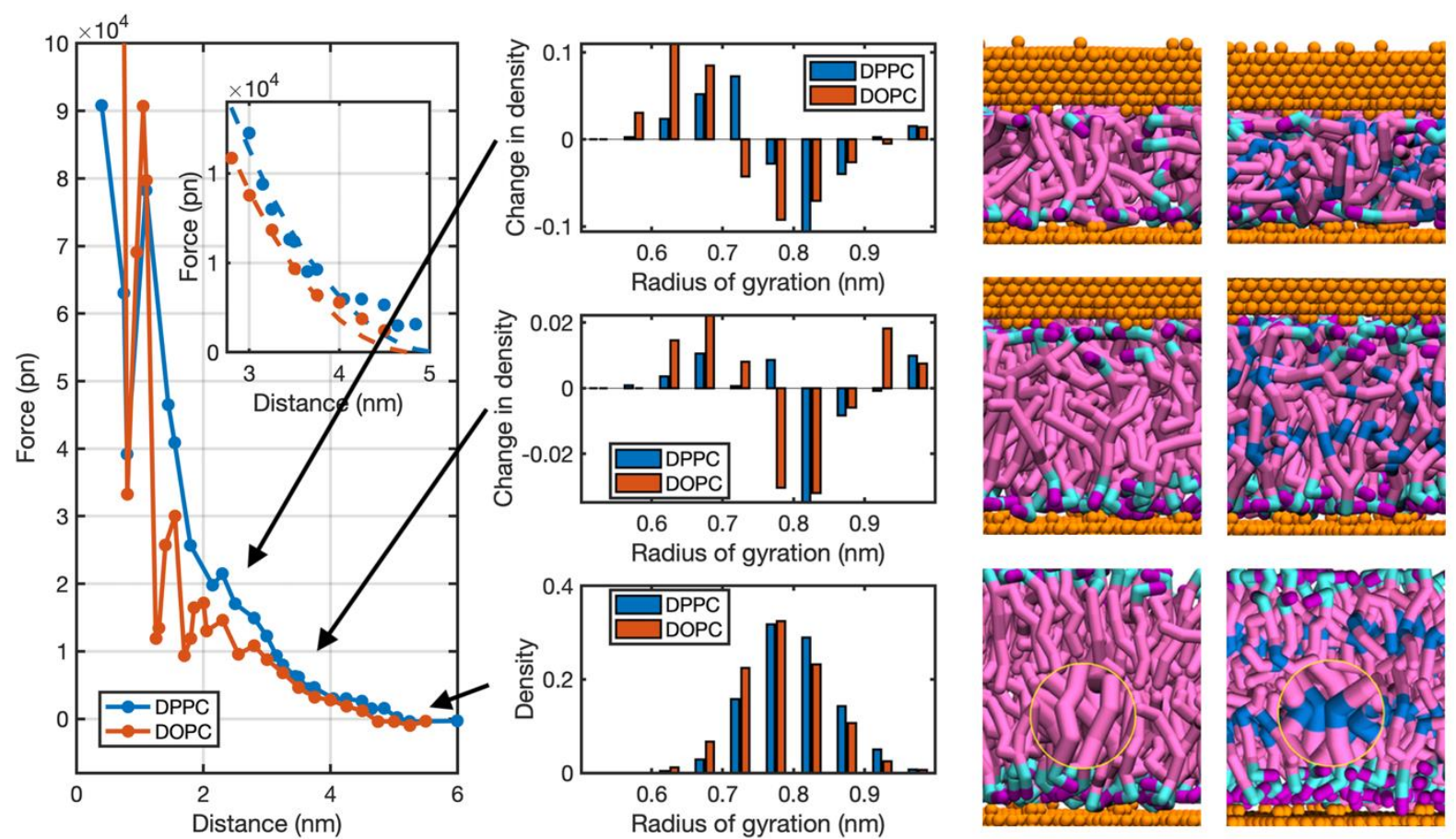

Figure 2: Simulated Force-distance curves for DOPC (orange curve) and DPPC (blue curve) SLBs; dashed lines, in the inset, are the modified Hertz fits, also used for fitting the AFM-FS curves on SLBs. Distribution of the radius of gyration for configurations extracted from the points indicated by the arrow and corresponding snapshots extracted from MD simulations. The blue segments indicate the coarse-grained beads (Martini C3 particle) describing the double-bond moiety in the structure of DOPC.

The simulated force-distance curves are qualitatively very similar to the ones obtained from the AFMFS measurements on SLBs. Moreover, the modified Hertz fit applied to the experimental force distance curves can still be used for describing the simulated ones with remarkable accuracy (see Fig. 2). The offset in the absolute value of computed forces with respect to the experiments is related to the details of the simulations (size and shape of the simulated mechanical probe, etc.).

In agreement with the experimental results, the DPPC SLB displays a stiffer mechanical response with respect to the DOPC SLB. At small indentations (tip-surface distances between 6 and $3 \mathrm{~nm}$ ), the SLBs undergo an essentially elastic deformation which only entails minimal perturbations to the equilibrium configuration for both DOPC and DPPC (see Fig. 2); differences in the response of the two simulated SLBs can be mainly ascribed to the different cohesive energies (hydrophobic interactions) of the bilayers. At larger indentations (tip-surface distances of $\sim 2.5 \mathrm{~nm}$ ) a qualitative difference emerges for the two considered lipid species. The stiffness of the DPPC SLB still exceeds the one of the DOPC SLB, as expected; however, starting from a tip-surface distance of about 2.5 $\mathrm{nm}$, the DOPC SLB undergoes a more evident structural rearrangement with respect to DPPC (see 
Fig. 2, right panels). This behavior can be related to the presence of saturated chains in the fatty acid moieties of DPPC, in contrast with the unsaturated chains of DOPC. These structural features are reproduced by the potential parameters used in simulations and lead to the observed differences in the mechanical properties of the two species considered. The occurrence of C-C double bonds in the unsaturated fatty acid chains of DOPC provides these molecules with a higher propensity to deform under a mechanical stress, with respect to those of DPPC (see Fig. 2). The magnitude of this local distortion can be also visualized when computing the average radius of gyration for the individual lipid molecules constituting the bilayer. In this context, the radius of gyration provides a measure of the linearity of the molecular structure. As shown in Fig. 2, the radius of gyration of DOPC molecules exhibits a significant drop at tip-surface distances below $2.5 \mathrm{~nm}$, signaling molecular deformations towards a coil-like structure. These deformations affect the structure of the whole bilayer and the resulting mechanical response, as evidenced by the kinks in the computed forcedisplacement curves for DOPC in the range between 1 and $2 \mathrm{~nm}$. Based on these results, differences in the mechanical behavior of the two considered SLBs, representative of gel and fluid phase bilayers, can be related to the interplay between intermolecular cohesion energy and intramolecular deformation. It is also worth noting that, at very large stress values, irreversible (plastic) structural deformations, falling beyond the range of elastic deformations considered by the employed model, occur. On a qualitative level, results from these simulations confirm that the different cohesion energies of fluid and gel phase lipids and the molecular structure can explain their different mechanical behaviour as observed by AFM-FS.

\section{A mechanical model for both fluid and gel phase vesicles}

The two most widely employed models for vesicles' mechanics are limited to the study of either fluid or gel phase bilayers. However, in the previous paragraphs, we have shown that the experimentally determined vesicle stiffness $\mathrm{K}$ is directly proportional to the SLB bending modulus $K_{S L B}$ irrespectively of the phase state of the constituent bilayers, thus suggesting that their mechanical behavior can be interpreted within a unified theoretical framework.

In order to gain more insights into the relationship between the mechanical responses of a lipid bilayer in its vesicular and SLB forms, we developed a simple model that allows separating the contributions of membrane elasticity and luminal pressure from the mechanical response of a fluid or gel phase lipid vesicle subjected to an applied perpendicular force. As schematized in Figure 2, we model an adsorbed lipid vesicle as a system of two springs in series, with spring constants $\mathrm{K}_{1}$ and $\mathrm{K}_{2}$. Spring $\mathrm{K}_{1}$ accounts for the mechanical response of the membrane, while $\mathrm{K}_{2}$ accounts for those phenomena arising as a consequence of the volume/surface variations induced by the indentation process (the most relevant being internal pressurization). 


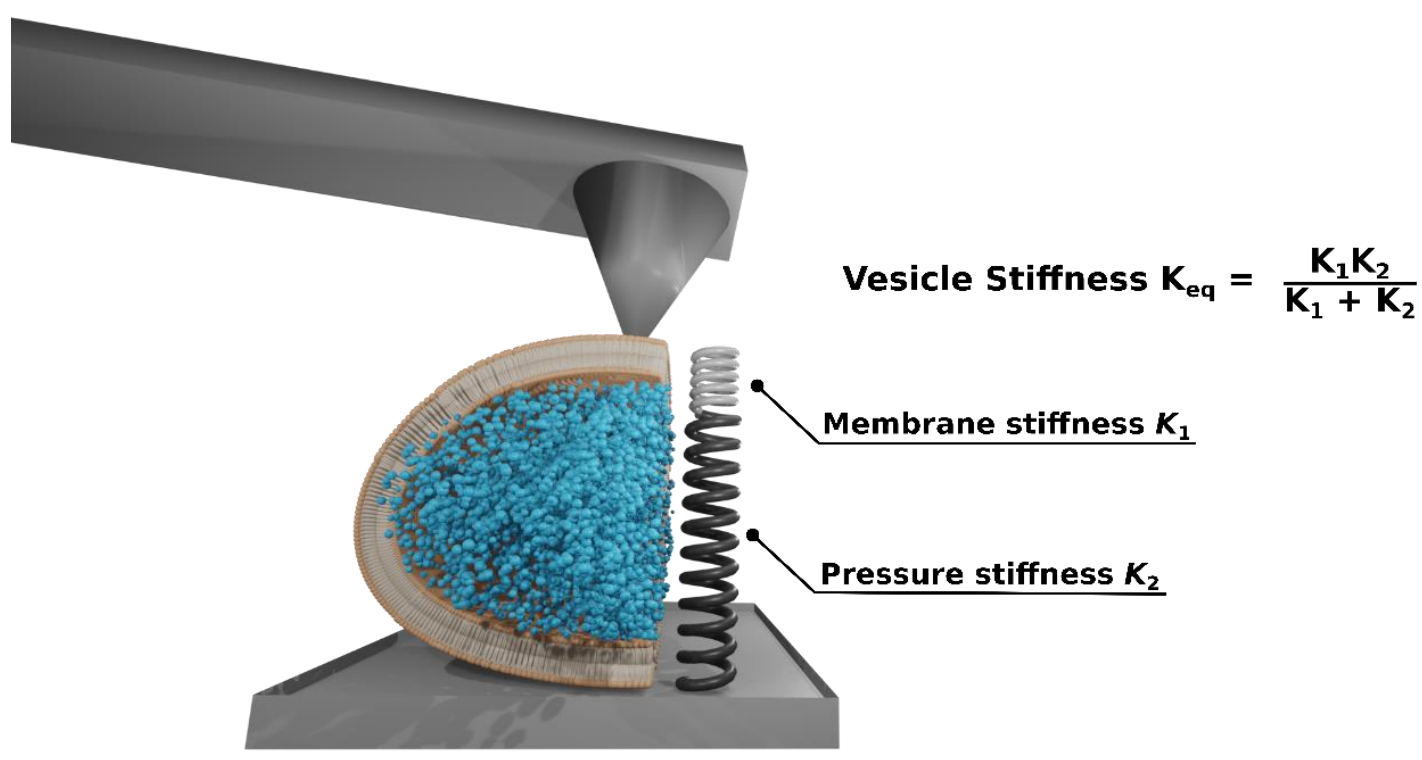

Figure 2: Spring-based model developed to describe the mechanical response of a vesicle to indentation. The stiffness of a vesicle accounts for the contributions of both membrane rigidity (mostly $\kappa$ ) and internal pressurization and can be described by a system of two springs in series. As a consequence, AFM-FS indentation experiments sample the overall spring constant of the whole system, which is lower than both the single spring constants $K_{1}$ and $K_{2}$.

More specifically, the slope observed in the linear part of the AFM force-distance curves represents the equivalent spring constant of the system, $K_{e q}$, which is related to $K_{1}$ and $K_{2}$ by Equation 3 .

$$
K_{e q}=\frac{K_{1} K_{2}}{K_{1}+K_{2}}
$$

The relation expressed by Equation 3 implies that the value of $K_{\text {eq }}$ is lower than the values of both $K_{1}$ and $K_{2}$. Another implication of the model is the different mechanical response to indentation of fluid and gel phase lipid vesicles. For very soft fluid phase vesicles, we can assume that $K_{1} \ll K_{2}$, hence obtaining $K_{e q} \sim K_{1}$, which means that the stiffness measured from the AFM force-distance curves $\left(K_{e q}\right)$ is close to the actual value of the membrane stiffness $\left(K_{1}\right)$. When dealing with stiffer membranes, as in the case of gel phase bilayers, $K_{1}$ increases and becomes comparable with $K_{2}$; in this new configuration, both springs deform (although to different extents) under application of a force. In this second scenario, the stiffness calculated from the AFM force-distance curves, $\left(K_{e q}\right)$ results from the combination of both springs (hence from the contributions of both membrane and luminal pressure).

\section{Thin Shell Theory underestimates the mechanical response of gel phase lipid vesicles}

As detailed above, TST models a vesicle as a hollow homogeneous shell with no internal pressure ${ }^{59}$. Reissner et al. ${ }^{21,22}$ derived a TST-based analytical solution (Equation 4) for describing the relation between force and shell indentation ${ }^{60}$ : 


$$
F=\left(\frac{4 E h^{2}}{R^{2} \sqrt{3\left(1-v^{2}\right)}}\right) \delta
$$

Where $E$ is the Young modulus, $h$ the bilayer thickness, $\delta$ the penetration depth, $R$ the shell (vesicle) radius of curvature and $v$ the Poisson modulus. Equation 4 is the analogous of Hooke's law, where the term within the parentheses represents, for small penetration depths, the stiffness of the vesicle. Once $K$ is known, $E$ and $\mathrm{K}$ can be determined, applying equation 2 from TST. Equation 4 is designed for hollow shells; hence it assumes that the stiffness (the proportionality constant between $F$ and $\delta$ ) is only ascribed to the vesicle membrane.

According to the just defined spring-based model, if we use the stiffness obtained from the AFM measurements, $K_{\text {eq }}$ (a combination of $K_{1}$ and $K_{2}$ ) to estimate the Young Modulus from Equation 4, we could obtain unexpected results. Notably, since $K_{e q}$ is lower than $K_{1}$, the mechanical contribution of the membrane and hence the values of $E$ and $\kappa_{V}$ are necessarily underestimated. Table 2 shows the bending moduli obtained by substituting $K_{\text {eq }}$ into the Reissner equation, for the panel of investigated liposomes and a comparison with the values of bending modulus obtained for the SLBs; the errors represent the uncertainties obtained by bootstrapping (1000 repetitions of 5 draws, with replacement).

Table 2

\begin{tabular}{|c|c|c|}
\hline & $\begin{array}{c}\text { Kv from TST } \\
\left(k_{B} T\right)\end{array}$ & $\begin{array}{l}K S L B \text { from modified } \\
\text { Hertz model }\left(k_{B} T\right)\end{array}$ \\
\hline DOPC & $18.7 \pm 1.8$ & $19.2 \pm 2.8$ \\
\hline POPC & $39.3 \pm 5.4$ & $47.6 \pm 5.6$ \\
\hline DPPC & $113.4 \pm 20.2$ & $240.1 \pm 50.5$ \\
\hline DSPC & $125.9 \pm 15.4$ & $335.8 \pm 48.1$ \\
\hline
\end{tabular}

As hypothesized, the $\kappa_{v}$ values obtained via TST from vesicle indentation are lower than the ones obtained via the modified Hertz model from the corresponding SLBs $\left(\kappa_{S L B}\right)$. Interestingly, the higher the vesicle stiffness, the higher the difference between $\kappa_{V}$ and $\kappa_{S L B}$. These results support the predictions of this spring model; indeed, when probing very soft liposomes, like DOPC, $K_{\text {eq }}-K_{1}$ and the Reissner formula yields results in good accord with the values measured on SLBs. However, as the $K_{1}$ of the probed liposomes increases and becomes proportional to $K_{2}$, the approximation is not valid anymore; $K_{e q}$ will be lower than the other two spring constants, yielding values of $K_{v}$ lower than the respective $K_{S L B}$.

\section{Estimating the membrane-associated spring constant}

Since the SLB indentation can be modeled as a 2D process, the complexity related to the 3D geometry of vesicles can be circumvented and the contributions from the vesicle internal pressure 
neglected. Moreover, since the literature on SLB mechanics is well-established and there are very few uncertainties regarding data interpretation, we herein assume that the $\kappa_{S L B}$ values are the ones that most closely represent the intrinsic bending rigidity of the membranes. Leveraging this assumption, if we now plug the $\kappa_{S L B}$ values back into (2) and (4), we can extract the correct values for the spring constant associated with the vesicle membrane, $K_{1}$. Table 3 displays the $K_{1}$ values that we obtained for the probed vesicles and compares them with the respective $K_{e q}$ values, calculated directly from the AFM force-distance curves.

Table 3

\begin{tabular}{cccc}
\hline & $\begin{array}{c}K_{1} \text { derived from } \\
\text { SLB } \\
\text { assumption } \\
(\mathbf{m N} / \mathbf{m})\end{array}$ & $\begin{array}{c}K_{\text {eq }} \text { from the AFM } \\
\text { curves }(\mathbf{m N} / \mathbf{m})\end{array}$ & $\begin{array}{c}K_{2} \text { obtained by } \\
\text { substituting } K_{1} \\
\text { and } \boldsymbol{K}_{\text {eq }} \text { in }(\mathbf{3}) \\
(\mathbf{m N} / \mathbf{m})\end{array}$ \\
\hline DOPC & $7.0 \pm 0.4$ & $6.0 \pm 0.6$ & $\sim 43.1$ \\
\hline POPC & $22.0 \pm 2.9$ & $14.5 \pm 1.5$ & $\sim 40.8$ \\
\hline DPPC & $74.4 \pm 7.2$ & $28.0 \pm 3.6$ & $\sim 44.9$ \\
\hline DSPC & $97.4 \pm 8.4$ & $34.0 \pm 3.3$ & $\sim 52.2$ \\
\hline
\end{tabular}

The results in Table 3 strongly support the predictions derived from the spring model; indeed, $K_{1}$ from DOPC, which was the softest probed liposome, is very close to $K_{\text {eq }}$ calculated from the AFM measurements. On the other hand, as the stiffness of the probed liposomes increases, their $K_{1}$ values start to differ more from their respective $K_{\text {eq. }}$. Using the newly obtained values of $K_{1}$, we can exploit Equation 3 to derive the values of $K_{2}$ for the different liposomes. These values are shown in the third column of Table 3 and should recapitulate the mechanical contribution arising from changes in the volume/surface of the vesicles, the most relevant being the internal pressurization. Surprisingly, the values of $K_{2}$ are very similar for most of the probed liposomes, irrespective of their membrane phase state. Only DSPC vesicles display a slightly increased pressurization, compared to the other ones. These results suggest that the mechanical contributions from the internal pressurization of most of the probed vesicles are very similar, across different liposome types. Looking at the $K_{2}$ values for the fluid phase liposomes (DOPC and POPC), it can be seen that the pressure plays the predominant role in the general mechanical response of these vesicles (with DOPC having a $K_{2}$ one order of magnitude higher than $K_{1}$ ), this is in perfect accord with findings by Vorselen et al. ${ }^{23}$, according to which the internal pressure of fluid phase liposomes provides the most relevant contribution to the vesicles mechanical response. The obtained results also highlight the different contributions of membrane elasticity and internal pressure to the overall vesicle stiffness, in fluid and gel phase liposomes; in the first ones, the greater contribution to the vesicle stiffness is given by the internal pressure while in the second ones, the contributions of membrane and pressure become comparable, and the vesicle mechanical response is given by their combination. 


\section{The difference between $K_{1}$ and $K_{2}$ could explain the slope change often observed in the second part of the AFM force-distance curves}

When the penetration depth is further increased (to values much larger than the membrane thickness), different events may take place: the AFM tip can puncture the bilayer (as often suggested by a sudden drop in the force signal in several vesicle indentation curves), the vesicles may lose part of their luminal content (depressurization) or even burst under excessive pressure. The manifestation of these events varies across the different vesicle types; from the analysis of the force curves obtained during the AFM-FS measurements on vesicles, we found that the ratio between $K_{1}$ and $K_{2}$ could provide an interpretation of the variability in the mechanical response characterizing the second part of the force-distance curves. Figure 3 displays the force curves collected during a representative ensemble of indentation cycles performed on the four different vesicle types (grey curves); the red curves are the average curves, obtained by averaging the value of the displayed grey curves at each separation point. As seen in Figure 3, the straightness of the red curves decreases from DOPC to DSPC, with the latter displaying a pronounced slope change.

These different behaviors can be rationalized by analyzing how $K_{e q}$ changes when the value of $K_{2}$ is decreased. For those cases in which the overall stiffness is dominated by $K_{2}, K_{e q} \sim K_{1}$, and hence a variation in $K_{2}$ has a negligible effect on the observed curve slope $\left(K_{e q}\right)$; since the new value of $K_{2}$ still largely exceeds $K_{1}$ (see Equation 3 ). On the other hand, when $K_{2}$ is comparable with $K_{1}$, its variation has a stronger impact on $K_{\text {eq }}$, generating an appreciable change in the slope of the curve. A decrease in $K_{2}$ could come as a consequence of larger indentations, which imply an increase in the internal pressurization of the vesicles, an event that could trigger the release of part of the internal fluid with a subsequent loss of volume. As a result, the vesicle would consequently have less fluid inside its lumen, hence being less pressurized, i.e. its $K_{2}$ would have a lower value. On the other hand, since $K_{1}$ represents the membrane stiffness, which should not be affected by depressurization phenomena, its value is assumed to remain constant during the whole indentation process. According to these predictions, in Figure 3 the effect of depressurization on the curves' slope is negligible for DOPC (the curves retain the initial slope also for larger indentations) but it becomes gradually more important approaching the stiffer gel phase liposomes, where the curves display larger variability for higher indentation values. The onset of a second linear regime in the forcedistance curves has been also recorded in the AFM studies of Vorselen et al. ${ }^{23}$ and Calò et al. ${ }^{12}$, while Vella et al. ${ }^{61}$ obtained a similar response from indentation tests on inflated spherical shells; most of these studies point at the pressure as the main responsible for the observed effect. This aspect might also explain why in stiffer liposomes, variations in the measured values of both stiffness and bending modulus are higher than the ones found for fluid phase ones. 

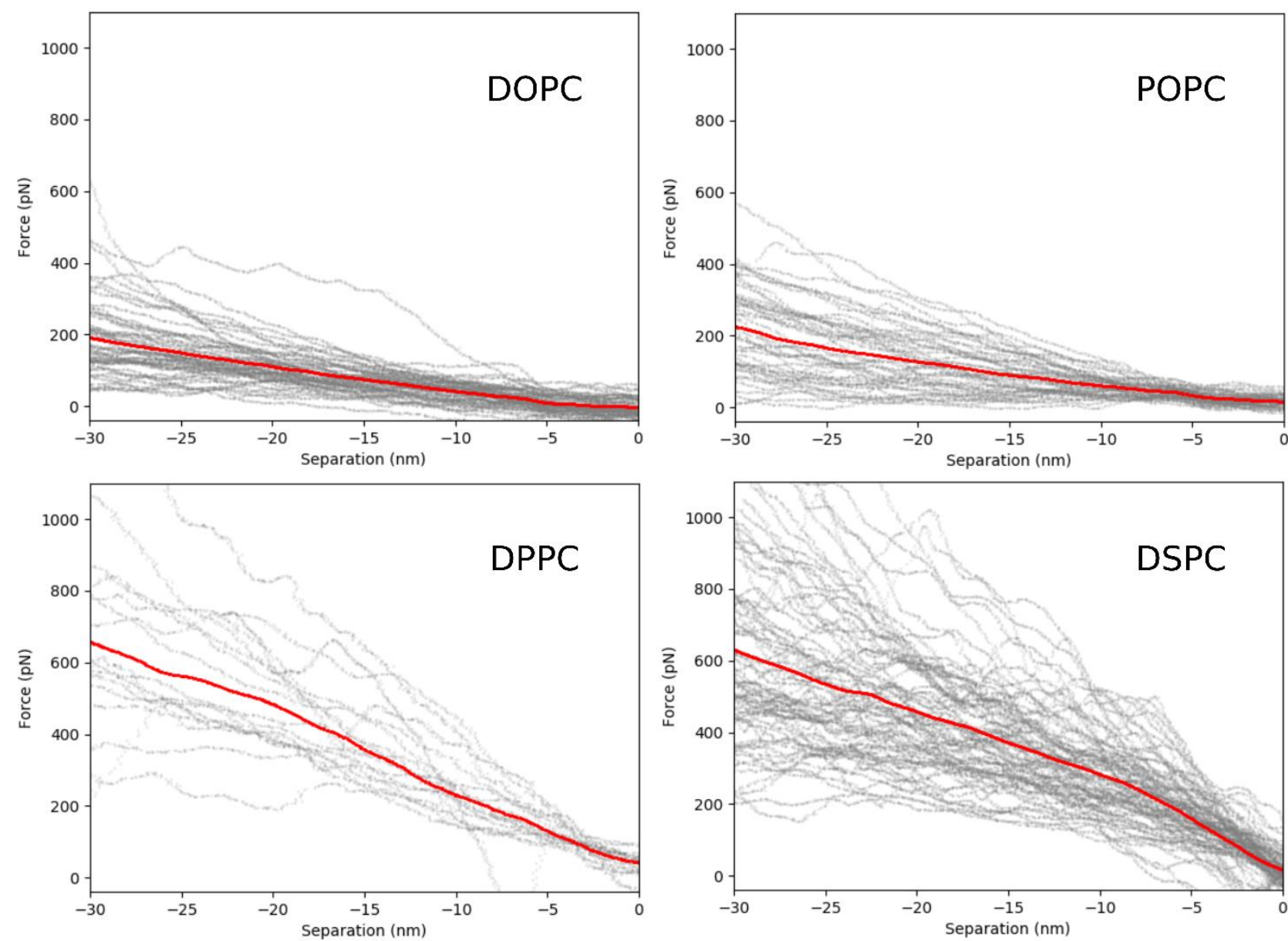

Figure 3: Average curves for the four different liposome types. Each average curve (red curve) was calculated by computing the average value of the single force-distance curves at each separation value. As can be seen, when going from DOPC to DSPC, $K_{1}$ and $K_{2}$ become comparable and variations in $K_{2}$ due to leakages of internal fluid during the indentation may have a stronger impact on $K_{e q}$ and on the observed curve slope.

\section{Conclusions}

Lipid membranes are involved in a plethora of relevant biological processes; for this reason, characterizing their mechanical properties can help understanding fundamental interactions between interfaces at the nanoscale. Force Spectroscopy techniques can be used to probe the mechanical properties of nanosized membranous envelopes such as vesicles, viruses, and other organelles; despite the high accuracy of these techniques, the data interpretation still stirs debate, ultimately leading to disagreement on the results obtained with different techniques.

By performing AFM-FS on a set of fluid and gel phase SLBs, we characterized their mechanical response in terms of bending modulus, which is an intrinsic descriptor of the membrane rigidity. Coarse-grained MD simulations, performed on realistic SLBs models confirmed and supported the AFM-FS results, showing that the differences, experimentally observed between fluid and gel phase bilayers, can be ascribed to the interplay between intermolecular cohesion energy and intramolecular deformation. 
Leveraging these results, we probed (by means of AFM-FS) the same set of fluid and gel phase lipid bilayers, in the liposome configuration, and found that the mechanical response of a lipid vesicle to an applied deformation can be modeled by a system of two springs in series. One of the springs accounts for the effect of membrane elasticity while the other for the effects arising from large volume/surface variations, whose greater contribution comes from the luminal pressure.

Exploiting this spring-based model, we find that despite not accounting for internal pressure contributions, the TST can still be used to extract the bending modulus values of very soft vesicles (like DOPC), for which the spring constant representing membrane stiffness has a negligible value compared to the one representing the luminal pressure. When these two contributions become comparable, the mechanical response of vesicles is a combination of the two springs and cannot be correctly analyzed by only means of TST. By assuming that the correct values of the bending modulus are the ones obtained from the AFM-FS on SLBs, we find that the pressurization of most of the probed vesicles (which had similar size) is similar, independently of the lipids forming the bilayer. Moreover, our mechanical model provides an interesting interpretation of the change in the slope displayed in the force curves of stiffer liposomes, showing that when the two spring constants are comparable, a change in the internal pressure would have a more appreciable effect on the vesicle stiffness, probed through AFM-FS.

Our findings contribute to shed light on the nanomechanics of lipid vesicles and provide a possible explanation to the discrepancy that is often observed among the results of bending modulus obtained from intact vesicles and SLBs. Future works will be aimed to quantify the extent of the internal pressure contribution, in order to correct and hence extend the applicability of TST based-models to the description of both gel and fluid phase vesicles. Ultimately, when applied to natural vesicles, such as EVs, the simple but clear-cut insights afforded by our model might help to better understand fundamental biological processes that involve vesicular deformation and/or reorganization.

\section{Acknowledgments}

This research has received funding from the Horizon 2020 Framework Programme under the grants FETOPEN-801367 "evFOUNDRY" and FETPROACT-EIC-05-2019 "Bio-Organic Wetsuits". We thank the SPM@ISMN research facility for support in the AFM experiments. 


\section{Bibliography}

1 G. Van Niel, G. D’Angelo and G. Raposo, Nat. Rev. Mol. Cell Biol., 2018, 19, 213-228.

2 I. Dulińska, M. Targosz, W. Strojny, M. Lekka, P. Czuba, W. Balwierz and M. Szymoński, J. Biochem. Biophys. Methods, 2006, 66, 1-11.

3 S. M. Hosseini and J. J. Feng, Biophys. J., 2012, 103, 1-10.

4 D. Vorselen, S. M. van Dommelen, R. Sorkin, M. C. Piontek, J. Schiller, S. T. Döpp, S. A. A. Kooijmans, B. A. van Oirschot, B. A. Versluijs, M. B. Bierings, R. van Wijk, R. M. Schiffelers, G. J. L. Wuite and W. H. Roos, Nat. Commun., 2018, 9, 4960.

B. Whitehead, L. P. Wu, M. L. Hvam, H. Aslan, M. Dong, L. DyrskjØt, M. S. Ostenfeld, S. M. Moghimi and K. A. Howard, J. Extracell. Vesicles, , DOI:10.3402/jev.v4.29685.

6 R. P. Rand and A. C. Burton, Biophys. J., 1964, 4, 115-135.

7 R. Dimova and K. A. Riske, in Handbook of Electroporation, 2017, vol. 1, pp. 235-252.

8 J. van Mameren, G. J. L. Wuite and I. Heller, in Methods in Molecular Biology, 2018, vol. 1665, pp. 3-23.

9 M. Krieg, G. Fläschner, D. Alsteens, B. M. Gaub, W. H. Roos, G. J. L. Wuite, H. E. Gaub, C. Gerber, Y. F. Dufrêne and D. J. Müller, Nat. Rev. Phys., , DOI:10.1038/s42254-018-0001-7.

10 P. Parisse, I. Rago, L. Ulloa Severino, F. Perissinotto, E. Ambrosetti, P. Paoletti, M. Ricci, A. P. Beltrami, D. Cesselli and L. Casalis, Eur. Biophys. J., 2017, 46, 813-820.

M. C. Piontek, R. B. Lira and W. H. Roos, Biochim. Biophys. Acta - Gen. Subj., 2019, 129486.

12 A. Calò, D. Reguera, G. Oncins, M. A. Persuy, G. Sanz, S. Lobasso, A. Corcelli, E. PajotAugy and G. Gomila, Nanoscale, 2014, 6, 2275-2285.

13 R. Sorkin, R. Huisjes, F. Bošković, D. Vorselen, S. Pignatelli, Y. Ofir-Birin, J. K. Freitas Leal, J. Schiller, D. Mullick, W. H. Roos, G. Bosman, N. Regev-Rudzki, R. M. Schiffelers and G. J. L. Wuite, Small, 2018, 14, 1-8.

14 O. Et-Thakafy, N. Delorme, C. Gaillard, C. Mériadec, F. Artzner, C. Lopez and F. Guyomarch, Langmuir, 2017, 33, 5117-5126.

15 Y. Takechi-Haraya, Y. Goda and K. Sakai-Kato, Langmuir, 2018, 34, 7805-7812.

16 P. B. Canham, J. Theor. Biol., 1970, 26, 61-81.

17 W. Helfrich, Zeitschrift für Naturforsch. C, 1973, 28, 693-703.

18 D. Boal, Mechanics of the cell, second edition, 2012.

19 L. D. Landau and E. M. Lifshitz, Theory of Elasticity, Third Edition: (Course of Theoretical Physics), 1986.

20 R. D. Gregory, T. I. Milac and F. Y. M. Wan, SIAM J. Appl. Math., 1998, 59, 1080-1097.

21 R. Eric, J. Math. Phys., 1912, 25, 80-85.

22 E. Reissner, J. Math. Phys., 1946, 25, 80-85.

23 D. Vorselen, F. C. Mackintosh, W. H. Roos and G. J. L. Wuite, ACS Nano, 2017, 11, 26282636. 
D. Vorselen, M. C. Piontek, W. H. Roos and G. J. L. Wuite, Front. Mol. Biosci., 2020, 7, 1-14.

R. Kalluri and V. S. LeBleu, Science (80-. )., , DOI:10.1126/science.aau6977.

R. P. Richter, R. Bérat and A. R. Brisson, Langmuir, 2006, 22, 3497-3505.

L. A. Clifton, R. A. Campbell, F. Sebastiani, J. Campos-Terán, J. F. Gonzalez-Martinez, S. Björklund, J. Sotres and M. Cárdenas, Adv. Colloid Interface Sci., 2020, 277, 102118.

L. Caselli, A. Ridolfi, J. Cardellini, L. Sharpnack, L. Paolini, M. Brucale, F. Valle, C. Montis, P. Bergese and D. Berti, Nanoscale Horizons, , DOI:10.1039/d1nh00012h.

S. M. Kanan, W. T. Y. Tze and C. P. Tripp, Langmuir, 2002, 18, 6623-6627.

A. Ridolfi, L. Caselli, C. Montis, G. Mangiapia, D. Berti, M. Brucale and F. Valle, J. Microsc., 2020, 00, 1-10.

J. L. Hutter and J. Bechhoefer, Rev. Sci. Instrum., 1993, 64, 1868-1873.

A. Ridolfi, M. Brucale, C. Montis, L. Caselli, L. Paolini, A. Borup, A. T. Boysen, F. Loria, M. J. C. van Herwijnen, M. Kleinjan, P. Nejsum, N. Zarovni, M. H. M. Wauben, D. Berti, P. Bergese and F. Valle, bioRxiv, 2019, 854539.

34 D. Nečas and P. Klapetek, Cent. Eur. J. Phys., 2012, 10, 181-188.

35 S. J. Marrink and D. P. Tieleman, Chem. Soc. Rev., 2013, 42, 6801-6822.

36 R. Alessandri, F. Grünewald and S. J. Marrink, Adv. Mater., n/a, 2008635.

37 A. Summerfield, M. Baldoni, D. V Kondratuk, H. L. Anderson, S. Whitelam, J. P. Garrahan, E. Besley and P. H. Beton, Nat. Commun., 2019, 10, 2932.

S. Plimpton, J. Comput. Phys., 1995, 117, 1-19.

L. Fernandez-Puente, I. Bivas, M. D. Mitov and P. Méléard, Epl, 1994, 28, 181-186.

D. Marsh, Chem. Phys. Lipids, 2006, 144, 146-159.

A. Ridolfi, M. Brucale, C. Montis, L. Caselli, L. Paolini, A. Borup, A. T. Boysen, F. Loria, M. J. C. Van Herwijnen, M. Kleinjan, P. Nejsum, N. Zarovni, M. H. M. Wauben, D. Berti, P. Bergese and F. Valle, Anal. Chem., 2020, 92, 10274-10282.

S. Li, F. Eghiaian, C. Sieben, A. Herrmann and I. A. T. Schaap, Biophys. J., 2011, 100, 637645.

H. Hertz, Gesammelte Werke, 1881, 171, 155-196 ST-Ueber die Berul`hrung fester elastis.

44 E. K. Dimitriadis, F. Horkay, J. Maresca, B. Kachar and R. S. Chadwick, Biophys. J., 2002, 82, 2798-2810.

A. Åkesson, T. Lind, N. Ehrlich, D. Stamou, H. Wacklin and M. Cárdenas, Soft Matter, 2012.

Z. V. Leonenko, E. Finot, H. Ma, T. E. S. Dahms and D. T. Cramb, Biophys. J., , DOI:10.1529/biophysj.103.036681.

47 P. Bilotto, M. Lengauer, J. Andersson, U. Ramach, L. L. E. Mears and M. Valtiner, Langmuir, 2019, 35, 15552-15563.

T. Charitat, E. Bellet-Amalric, G. Fragneto and F. Graner, Eur. Phys. J. B, , 
DOI:10.1007/s100510050725.

49 J. Daillant, E. Bellet-Amalric, A. Braslau, T. Charitat, G. Fragneto, F. Graner, S. Mora, F. Rieutord and B. Stidder, Proc. Natl. Acad. Sci. U. S. A., 2005, 102, 11639-11644.

50 Y. Liu and J. F. Nagle, Phys. Rev. E - Stat. Physics, Plasmas, Fluids, Relat. Interdiscip. Top., , DOI:10.1103/PhysRevE.69.040901.

51 Z. A. Levine, R. M. Venable, M. C. Watson, M. G. Lerner, J. E. Shea, R. W. Pastor and F. L. H. Brown, J. Am. Chem. Soc., 2014, 136, 13582-13585.

52 L. Picas, F. Rico and S. Scheuring, Biophys. J., 2012, 102, L01-L03.

53 R. Dimova, Adv. Colloid Interface Sci., 2014, 208, 225-234.

54 J. F. Nagle, Chem. Phys. Lipids, 2017.

55 J. Henriksen, A. C. Rowat, E. Brief, Y. W. Hsueh, J. L. Thewalt, M. J. Zuckermann and J. H. Ipsen, Biophys. J., , DOI:10.1529/biophysj.105.067652.

56 Z. Yi, M. Nagao and D. P. Bossev, J. Phys. Condens. Matter, 2009, 21, 1-7.

57 N. Delorme and A. Fery, Phys. Rev. E - Stat. Nonlinear, Soft Matter Phys., , DOI:10.1103/PhysRevE.74.030901.

58 R. M. Hochmuth, J. Y. Shao, J. Dai and M. P. Sheetz, Biophys. J., 1996, 70, 358-369.

59 M. LeClaire, J. Gimzewski and S. Sharma, Nano Sel., 2020, 1-15.

60 J. D. Berry, S. Mettu and R. R. Dagastine, Soft Matter, 2017, 13, 1943-1947.

61 D. Vella, A. Ajdari, A. Vaziri and A. Boudaoud, J. R. Soc. Interface, 2012, 9, 448-455. 